\title{
Seismic scattering for a point scatterer -with a special interest to the application in full waveform inversion-
}

\author{
Hitoshi Mikada \\ Dept. of Civil and Earth Res. Eng., Kyoto University
}

\begin{abstract}
We revisit a seismic scattering as a key physical process to be considered in the processing of multiparameter seismic full waveform inversion (FWI). FWI implicitly uses seismic scattering theories based on the assumption that the contrast in seismic velocities is weak at each location of medium inhomogeneities since the fundamental equation of FWI comes from the perturbation theory. Since each perturbed location is considered as a point elastic scatterer, we try to relax the assumption of low contrast in elastic parameters at the scatterers by means of elastic scattering theories. In mathematics, a "point" does not have any volume so that it could not have any elastic parameters. We therefore start with a homogeneous spherical inclusion as a heterogeneous volume that has arbitrary contrast to the surrounding medium. The problem is nothing but a Rayleigh scattering of seismic waves that has exact solutions to incident compressional and shear waves without any assumption in the spherical inclusion case. After taking the smallest limit of the spherical inclusion, scattered waves are expressed by a polynomial of the finite number of terms with the Dirac's delta function for incoming both compressional and shear waves to the point scatterer. Our results show that the scattered waves are similar to what has been obtained for a weak contract case but differs in terms of the combination of elastic parameters. Based on our results, we think our solution to the inhomogeneous wave equation could directly be applied to FWI for arbitrary contrast in elastic parameters like that from the conventional perturbation theory for weak contrast case.
\end{abstract}

\section{Introduction}

In the current practice of full waveform inversion, seismic velocities are the primary objectives of data analysis stated: "the main objectives of full waveform inversion (FWI) is to build a highresolution velocity model." ${ }^{1)}$ Seismic velocities are influenced by the other dynamic parameters such as elastic moduli and density, etc., of materials and the extension of FWI to coommodate the influence of density $^{2,3)}$ has recently been tried, too. The choice of parameters and processing flow is a key in these multi-parameter $\mathrm{FWI}^{1-3)}$, it has been reported necessary to maneuver the path to the optimum minimum in the presense of local minima in the multi-parameter least-squares norm space. The fundamental equations for FWI have been proposed in the early pioneer work using the perturbation theory ${ }^{4,5)}$. The perturbation theory assumes weak heterogeneity or low contrast in elastic properties, i.e., low-contrast condition between anomalous bodies and the surroung media. The anomalous bodies become secondary sources of seismic waves in response to incident seismic waves. The physical process is called seismic scattering caused by the heterogeneous anomalous materials. Scatterred seismic waves have been used as signals generated at bodies whose seismic velocities are different from the surrounding media.

Researches on FWI now move on to more complex structure using elastic properties of subsurface material in complex structure that may not assure the low-contrast condition. Arbitrary contrast condition needs to be considered in FWI to deal with really "heterogeneous" anomalies that could exist in the subsurface. For future application of FWI, Also, as far as the parameters being directly estimated in FWI are seismic compressional and shear velocities, the difference in the radiation pattern of scattered waves caused by these velocities may not be well exploited since these velocities are interrelated through intervening varibles such as elastic moduli and density. Compressional and shear velocities are mathematically not independent from each other. It is, therefore, necessary to consider the dependency of parameters being directly estimated in FWI as well as the arbitrary condition.

In this paper, we try to accommodate both the arbitrary condition and the parameters indipendent from each other. For these objectives, we revisit a 
seismic scattering problem for a spherical inclusion of heterogeneous material in a homogeneous medium $^{6}$. We found the exact solutions to the scattering problem could be used as a Green's function to a point scatterer of arbitrary condition after taking the smallest limit of the radius of the sphere ${ }^{7)}$. These Green's functions satisfies inhomogeneous Helmholtz equations and could directly be applied to the Rayleigh-Sommerfeld Integral equation that are commonly used in seismic migration, diffraction tomography, or any other back-propagation techniques. The equation could readily extended to multiple scattering problems using a Born series.

\section{Elastic Scattering}

For an elastic medium that includes a spherical heterogeneous scatterer at the origin of the spherical coordinate system $(r, \theta, \varphi)$, an inhomogeneous wave equation in the frequency domain is expressed as follows in the elastic medium outside of the inclusion for incident elastic waves.

$$
\begin{aligned}
& \left(\nabla^{2}+k_{P}^{2}\right) \phi(\vec{r})=k_{P}^{2} A_{P}(\vec{r}) \phi(\vec{r})+k_{P}^{2} B_{P}(\vec{r}) \psi(\vec{r}) \\
& \left(\nabla^{2}+k_{S}^{2}\right) \psi(\vec{r})=k_{S}^{2} A_{S}(\vec{r}) \phi(\vec{r})+k_{S}^{2} B_{S}(\vec{r}) \psi(\vec{r})
\end{aligned}
$$

where $\vec{r}, \phi$ and $\psi$ denote the position vector in the spherical coordinate system, a scaler displacement potential and one of the components of a vector displacement potential, respectively. $k_{P}$ and $k_{S}$ are the wavenumbers for compressional and shear waves, respectively. $A_{P}, A_{S}, B_{P}$ and $B_{S}$ are the functionals to express the interaction of incident waves and the spherical scatterer.

We assume the bulk modulus $\kappa$, two Lame's constants, $\lambda$ and $\mu$, and the density to the elastic medium, and the contrasts to these parameters to be $\Delta \kappa, \Delta \lambda, \Delta \mu$, and $\Delta \rho$, respectively, to the spherical inclusion.

When the radius $a$ of the spherical scatterer is small, i.e., $k_{P} a \ll 1$ and $k_{S} a \ll 1$, compared to the wavelength of both compressional and shear waves incident to the inclusion, the solutions of eq.(1) have been obtained ${ }^{6,7)}$ to represent the Rayleigh scattering. Two series solutions to eq. (1) have the following form.

$$
\begin{aligned}
& \phi(\vec{r})=\phi^{(0)}(\vec{r})+\sum_{n=1}^{\infty} \phi^{(n)}(\vec{r}) \\
& \psi(\vec{r})=\psi^{(0)}(\vec{r})+\sum_{n=1}^{\infty} \psi^{(n)}(\vec{r})
\end{aligned}
$$

The first order Born approximation simplifies eq. (2) to the following equations.

$$
\begin{aligned}
& \phi(\vec{r})=\phi_{0}(\vec{r})+\phi^{(1)}(\vec{r}) \\
& \psi(\vec{r})=\psi_{0}(\vec{r})+\psi^{(1)}(\vec{r})
\end{aligned}
$$

In eq. (3), $\phi^{(0)}(\vec{r})$ and $\psi^{(0)}(\vec{r})$ represent incident compressional and shear waves, while $\phi^{(1)}(\vec{r})$ and $\psi^{(1)}(\vec{r})$ single scattered. Taking the smallest limit of the radius, we get an equivalent body force component to each contrast in the elastic moduli and in density.

\section{(1) A plane compressional wave incidence}

The scattered waves from the point scatterer located at the origin of the homogeneous medium could be obtained from the following inhomogeneous wave equations for a plane compressional wave, i.e., $\psi^{(0)}(\vec{r})=0$, travelling in a single direction.

$$
\begin{aligned}
& \left(\nabla^{2}+k_{P}^{2}\right) \phi^{(1)}(\vec{r})=k_{P}^{2} S_{P}^{P} \delta(\vec{r}) \\
& \left(\nabla^{2}+k_{S}^{2}\right) \psi^{(1)}(\vec{r})=k_{S}^{2} S_{S}^{P} \delta(\vec{r})
\end{aligned}
$$

where $S_{P}^{P}$ and $S_{S}^{P}$ denote the magnitudes of the equivalent body forces that generate scattered wavefields, and are expressed in the following equations. For simplicity, we assume the place wave travels in $\mathrm{z}$ direction in 3D Cartesian coordinates $(x, y, z)$.

$$
\begin{aligned}
& S_{P}^{P}=-\sum_{n=0}^{2}(-1)^{n} \frac{(2 n-1) ! !}{n ! k_{P}^{n}} C_{n}^{P}\left(\frac{\partial}{\partial z}\right)^{n} \phi_{0}(0) \\
& S_{S}^{P}=-\sum_{n=1}^{2}(-1)^{n} \frac{(2 n-1) ! !}{n ! k_{S}^{n}} C_{n}^{S}\left(\frac{\partial}{\partial z}\right)^{n} \phi_{0}(0)
\end{aligned}
$$

where

and

$$
\begin{gathered}
C_{0}^{P}=\frac{\Delta \kappa}{\lambda+2 \mu+\Delta \kappa} \\
C_{1}^{P}=\frac{\Delta \rho}{\rho} \\
C_{2}^{P}=\frac{\Delta \mu}{\lambda+2 \mu+\frac{2 \Delta \mu}{5 \mu}(\kappa+2 \mu)}
\end{gathered}
$$

$$
\begin{aligned}
& C_{1}^{S}=\frac{k_{S}^{2}}{k_{P}^{2}} C_{1}^{P} \\
& C_{2}^{S}=\frac{k_{S}^{3}}{k_{P}^{3}} C_{2}^{P}
\end{aligned}
$$

Two functions $S_{P}^{P} \delta(\vec{r})$ and $S_{S}^{P} \delta(\vec{r})$ indicates the secondary source located at the point scatterer of arbitrary contrast in elastic parameters for a plane compressional wave incidence. The body forces representing the above 5 coefficients are shown diagrammatically in Fig. 1. Finally, the scattered 
wavefields are expressed by the following equations.

$$
\begin{aligned}
& \phi^{(1)}(\vec{r})=-i \frac{k_{P}^{3} \phi^{(0)}(0)}{4 \pi}\left\{\sum_{n=0}^{2} C_{n}^{P} h_{n}^{(1)}\left(k_{P} r\right) P_{n}(\cos \theta)\right\} \\
& \psi^{(1)}(\vec{r})=-i \frac{k_{S}^{3} \phi^{(0)}(0)}{4 \pi}\left\{\sum_{n=1}^{2} C_{n}^{S} h_{n}^{(1)}\left(k_{S} r\right) P_{n}(\cos \theta)\right\}
\end{aligned}
$$

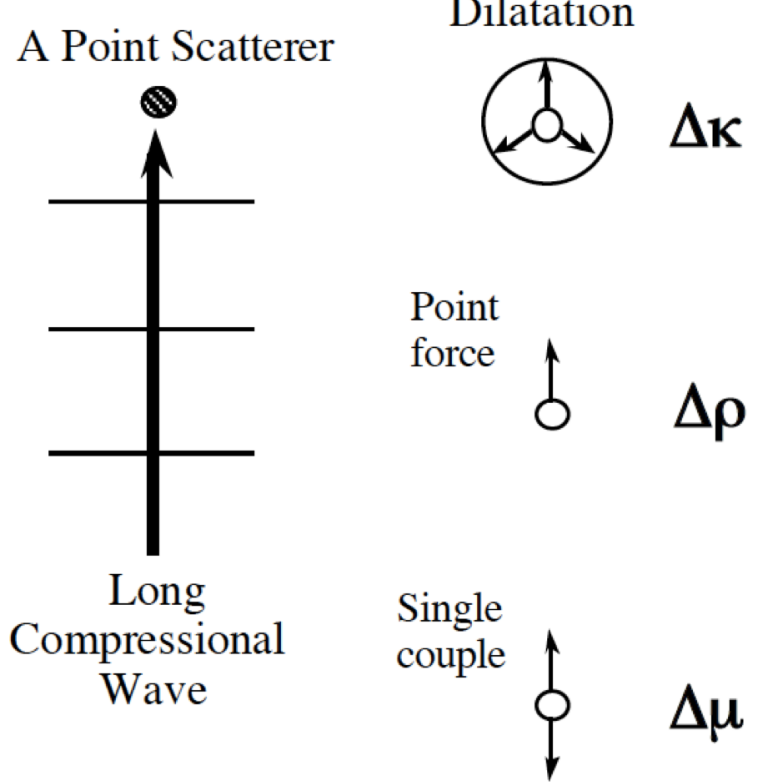

Fig.1 Equivalent body forces acting to a point scatterer for the incidence of a plane compressional wave. Three different types of the forces are caused by the contrast of indipendent elastic parameters ${ }^{7}$.

\section{A Point Scatterer}

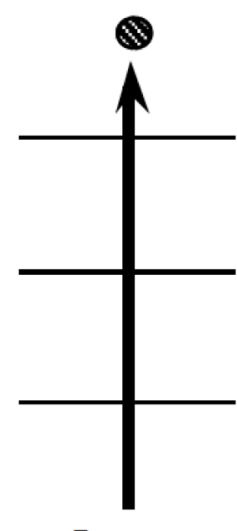

Long

Shear Wave

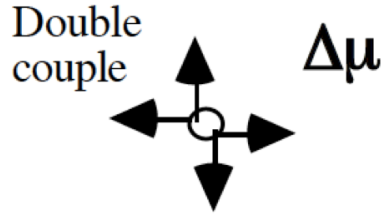

where $h_{n}^{(1)}$ and $P_{n}$ are the spherical Bessel function of the first kind and the associated Legendre function, respectively, for the $\mathrm{n}$-th order.

\section{(2) A plane shear wave incidence}

The scattered waves from the point scatterer located at the origin of the homogeneous medium could also be obtained from the following inhomogeneous wave equations for a plane shear wave, i.e., $\phi^{(0)}(\vec{r})=0$, travelling in $z$ direction.

$$
\begin{aligned}
& \left(\nabla^{2}+k_{P}^{2}\right) \phi^{(1)}(\vec{r})=k_{P}^{2} S_{P}^{S} \delta(\vec{r}) \\
& \left(\nabla^{2}+k_{S}^{2}\right) \psi^{(1)}(\vec{r})=k_{S}^{2} S_{S}^{S} \delta(\vec{r})
\end{aligned}
$$

where $S_{P}^{P}$ and $S_{S}^{P}$ denote the magnitudes of the equivalent body forces that generate scattered wavefields. Given that the incident shear wave is polarized in $x$ direction, the scattered wavefields are expressed in the following equations.

$$
\begin{aligned}
& S_{P}^{S}=-\sum_{n=1}^{2}(-1)^{n} \frac{(2 n-1) ! !}{n(n+1) \cdot n ! k_{S}^{n}} D_{n}^{P}\left(\frac{\partial}{\partial x}\right)\left(\frac{\partial}{\partial z}\right)^{n-1} \psi_{0}(0) \\
& S_{S}^{S}=-\sum_{n=1}^{2}(-1)^{n} \frac{(2 n-1) ! !}{n(n+1) \cdot n ! k_{S}^{n}} D_{n}^{S}\left(\frac{\partial}{\partial x}\right)\left(\frac{\partial}{\partial z}\right)^{n-1} \psi_{0}(0)
\end{aligned}
$$

where

and

$$
\begin{aligned}
& D_{1}^{P}=C_{1}^{P} \\
& D_{2}^{P}=C_{2}^{P}
\end{aligned}
$$

$$
\begin{aligned}
& D_{1}^{S}=C_{1}^{S} \\
& D_{2}^{S}=C_{2}^{S}
\end{aligned}
$$

Two functions $S_{P}^{S} \delta(\vec{r})$ and $S_{S}^{S} \delta(\vec{r})$ indicates the secondary source located at the point scatterer of arbitrary contrast in elastic parameters for a plane shear wave incidence. The body forces representing the above 4 coefficients in eq. (7) are shown diagrammatically in Fig. 2. Scattered wavefield could be estimated as for the compressional wave incidence.

$$
\begin{aligned}
& \phi^{(1)}(\vec{r})=-i \frac{k_{P}^{3} \psi^{(0)}(0)}{4 \pi}\left\{\sum_{n=0}^{2} D_{n}^{P} h_{n}^{(1)}\left(k_{P} r\right) P_{n}^{1}(\cos \theta) \cos \varphi\right\} \\
& \psi^{(1)}(\vec{r})=-i \frac{k_{S}^{3} \psi^{(0)}(0)}{4 \pi}\left\{\sum_{n=1}^{2} D_{n}^{S} h_{n}^{(1)}\left(k_{S} r\right) P_{n}^{1}(\cos \theta) \cos \varphi\right\}
\end{aligned}
$$

The advantage of these functions is that each term in each of eq. (5) and (7) is orthogonal to the other term(s) because of the orthogonality of the associated Legendre function. 


\section{Multiple scattering}

We started with the series solution in eq. (2) to solve the inhomogeneous equation in eq. (1). For simplicity, we first introduced the first order Born approximation that is equivalent to the assumption of single scattering. When multiple scattering process is considered, the scattering equations in the above should be extended as follows.

A general form of the multiple scattering is given in eq. (1). Substituting the potentials in eq. (1) with eq. (2), we may obtain recursive formula for the multiple scattering process with the following set of equations under the assumption of $\left\|\phi^{(m-1)}\right\| \gg$ $\left\|\phi^{(m)}\right\|$ and $\left\|\psi^{(m-1)}\right\| \gg\left\|\psi^{(m)}\right\|$.

$$
\begin{aligned}
& \left(\nabla^{2}+k_{P}^{2}\right) \phi^{(m)}(\vec{r})=k_{P}^{2} A_{P}(\vec{r}) \phi^{(m-1)}+k_{P}^{2} B_{P}(\vec{r}) \psi^{(m-1)}(\vec{r}) \\
& \left(\nabla^{2}+k_{S}^{2}\right) \psi^{(m)}(\vec{r})=k_{S}^{2} A_{S}(\vec{r}) \phi^{(m-1)}+k_{S}^{2} B_{S}(\vec{r}) \psi^{(m-1)}(\vec{r})
\end{aligned}
$$

where $m$ is the order of scattering or the Born series. The above equations would be solved using Rayleigh-Sommerfeld integral equation of the form hereafter.

$$
\begin{aligned}
& \phi^{(m)}(\vec{r})=-\oiiint_{r_{0} \in V} G\left(\vec{r}_{0}, \vec{r}\right) Y_{P}^{(m-1)}\left(\vec{r}_{0}\right) d \vec{r}_{0} \\
& \psi^{(m)}(\vec{r})=-\oiiint_{r_{0} \in V} G\left(\vec{r}_{0}, \vec{r}\right) Y_{S}^{(m-1)}\left(\vec{r}_{0}\right) d \vec{r}_{0}
\end{aligned}
$$

where

$Y_{P}^{(m-1)}(\vec{r})=k_{P}^{2} A_{P}(\vec{r}) \phi^{(m)}+k_{P}^{2} B_{P}(\vec{r}) \psi^{(m)}(\vec{r})$

$Y_{S}^{(m-1)}(\vec{r})=k_{S}^{2} A_{S}(\vec{r}) \phi^{(m)}+k_{S}^{2} B_{S}(\vec{r}) \psi^{(m)}(\vec{r})$

The convergence of the series would be assured if and only if the assumption of higher order scattering being of lower magnitude and of numerical stability.

\section{Discussion}

FWI is a technique that draws the most attention from exploration geophysicists as a method of highresolution velocity estimation using observed seismic signals. Wave propagation is one of the major physical processes frequently exploied to visualize invisible structure in exploration geophysics. Reflection, defraction, refraction or transmission of waves in the suvsurface could bring additively the knowledge on both kinematic and dynamic parameters of materials where the waves pass through. FWI has been developed based on the perturbation theory that is nothing but seismic scattering due to inhomogeneties. The effects of the wave scattering have been of interest not only to locate the location but to estimate the physical properties of inhomogeneities of the medium in the subsurface. The theory should be the same and could be unified for both FWI and seismic scattering ${ }^{13)}$. The radiation patterns of scattered waves to incident compressional and shear waves were estimated including arbitrary contrast in the parameters of elastic moduli and density for the inclusion of finite volume, for example a sphere ${ }^{4,6)}$.

The first order Born or Rylov approximation is used to solve the inhomogeneous Helmholtz equation under the assumption of weak inhomogeneites, i.e., implicit low-contrast condition with two Lame's constants and density. When the order of such inhomogeneities increases, approximation formula proposed in the thick literature based on the assumption of weak contrast may break up, and we need to think about arbitrary contrast to the inhomogeneities in the medium while keeping the energy of scattered waves smaller than that of the incident. The introduction of the Rayleigh scattering seems a good starting point in this regard.

Although the radiation pattern of secondary seismic source has been discussed ${ }^{1-4,9,10)}$, the orthogonality of spherical Legendre function has not been well recognized since the perturbation theory, which founded the basis of FWI, may not provide the patterns orthogonal to each other. The utilization of spherical inclusion or the Rayleigh scattering enables the use of equivalent body forces that generate wavefield orthogonal to each other after the integration over a closed surface surrounding the location of the forces.

There is discussion how to accommodate multiple scattering processes ${ }^{1,10)}$ as the enery of scattered energy becomes non-negligible compared to the incident. Although seismic migration or the other diffraction tomography techniques have been developped on the basis of single scattering theories, the assumption of single scattering could be easily violated depending on the frequency contents of seismic signals and the scattering intensity. The situation to raise the frequency contents to higher part could take place when trying to enhance the resolution in structural studies. In these cases, the problem of multiple scattering must be considered.

Multiple scattering theories on seismic waves have extensively studied and was reported as an important process in forming waveforms or seismic envelope $^{11,12)}$ in the analyses of short wavelength seismic waveforms. The importance of multiple scattering processes is clear to seek a tie between wave-theoretical exploration schemes and the analyses of the above short-wavelength multiple 
scattering theories.

\section{Conclusions}

We have driven body forces that represent elastic scattering phenomena for a "point" scatterer of arbitrary contrast in elastic parameters to the surround medium. We started from the spherical inclusion of heterogeneous material and took the smallest limit of the radius of the sphere. Our results showed that the scattering waves are caused by the following body forces: a dilatational source, a point force, and a single couple for an incident compressional plane wave, and a point force and a double couple for an incident shear plane wave.

We then formulated multiple scattering after introducing higher order terms of Born series.

\section{REFERENCES}

1) Alkhalifah, T., 2014, Full waveform inversion in an anisotropic world, EAGE Publ., 200pp., ISBN 978-90-73834-83-5.

2) Plessix, R.-E., Stopin, A., Milcik, P., and Matson, K., 2014, Acoustic and anisotropic multiparameter seismic full waveform inversion case studies, SEG Technical Program Expanded Abstracts 2014: pp. 1056-1060. doi: 10.1190/segam2014-0646.1

3) Bai, J., and Yingst, D., 2014, Simultaneous inversion of velocity and density in time-domain full waveform inversion. SEG Technical Program Expanded Abstracts 2014: 922-927. doi: 10.1190/segam2014-0532.1

4) $\mathrm{Wu}, \mathrm{R}$. , and Aki, K., 1985, Scattering characteristics of elastic heterogeneity, Geophysics, $50 \quad$ (4), 582-595. doi: 10.1190/1.1441934

5) Tarantola, A., 1986, A strategy for nonlinear elastic inversion of seismic reflection data." Geophysics, 51(10), 1893-1903. doi: 10.1190/1.1442046

6) Korneev, V.A., and Johnson, L.R., 1993, Scattering of elastic waves by a spherical inclusion-I. Limitations of asymptotic solutions, Geophys. J. Int., 115 (1), 251-263. doi: 10.1111/j.1365-246X.1993.tb05602.x

7) Mikada, H., Watanabe, H., and Sakashita, S., 1997, Evidence for subsurface magma bodies beneath Izu-Oshima volcano inferred from a seismic scattering analysis and possible interpretation of the magma plumbing system of the 1986 eruptive activity, Phys. Earth Planet. Inter, 104 (1-3), 257269. doi:10.1016/S0031-9201(97)00060-5

8) Mikada, H., 2004, Scattering Processes applied to Structural Interpretation, Proc. 3rd VAST-Hanoi University of Mining and Geology-Japan Joint Workshop, September 13-14, 2004, Hanoi,
Vietnam, 23-28.

9) Tarantola, A., 1986, A strategy for nonlinear elastic inversion of seismic reflection data, Geophysics, 51(10), 1893-1903. doi: 10.1190/1.1442046

10) Virieux, J., and Operto, S., 2009, An overview of full-waveform inversion in exploration geophysics, Geophysics, 74(6), WCC1-WCC26. doi: 10.1190/1.3238367

11) Mehta, C. H. (1983). Scattering theory of wave propagation in a two-phase medium. Geophysics, 48 (10), 1359-1370. doi: 10.1190/1.1441416

12) $\mathrm{Wu}$, R.-S., \& Aki, K. (1985). Elastic wave scattering by a random medium and the smallscale inhomogenieties in the lithosphere. $J$. Geophys. Res., 90 (B12), 10261-10273. doi: 10.1029/JB090iB12p10261

13) Teranishi, K., Mikada, H., and Takekawa, J., 2016, Preconditioning Elastic Full Waveform Inversion by Scattering Theory, Proc. EAGE Workshop on Velocities: Reducing Uncertainties in Depth, Kuala Lumpur, 25 April 2016. doi: 10.3997/2214-4609.201600077 